\title{
Matrix metalloproteinase activity in equine synovial fluid: influence of age, osteoarthritis, and osteochondrosis
}

\author{
P A J Brama, J M TeKoppele, B Beekman, P R van Weeren, A Barneveld
}

\begin{abstract}
Objective-To investigate the influence of age, osteoarthritis (OA), and osteochondrosis (OC) on the matrix metalloproteinase (MMP) activity in the synovial fluid (SF) of equine joints.

Methods-SF was collected from normal and osteoarthritic metacarpophalangeal joints (normal: 14 adult, 28 juvenile; OA: 22 adult). And from normal and osteochondrotic tarsocrural joints (5 months: 11 normal, 8 OC; 11 months: 7 normal, 6 OC). Subsequently, overall MMP activity was measured.

Results-The level of active MMPs was almost twofold higher in SF from juvenile horses (age up to 11 months) than in SF from mature animals (4-30 years; $\mathrm{p}<0.001$ ). In juvenile horses MMP activity was higher in 5 month old foals than in 11 month old foals $(p<0.01)$. In adult horses MMP activity was independent of age. In OA joints the activity was nearly twice as high as in normal joints $(p<0.001)$. In OC joints MMP activity was not significantly different from normal, age matched, control joints.

Conclusions-MMP activity in SF from normal adult joints is not related to age. In juvenile joints MMP activity is significantly higher than activity in joints from adult animals. It is hypothesised that the gradual decrease in MMP activity with increasing age reflects the declining metabolic activity resulting from ceasing growth and the accompanying decrease in cartilage remodelling. The increased MMP activity in osteoarthritic joints most likely reflects matrix destruction. In osteochondrosis MMP mediated matrix degradation appears not to be different from normal joints.

(Ann Rheum Dis 1998;57:697-699)
\end{abstract}

Labora

TNO Prevention and

Health, Division of

Vascular and

Connective Tissue

Research, Leiden,

the Netherlands

J M TeKoppele

B Beekman

Correspondence to:

P A J Brama, Department of

General and Large Animal

Surgery, Faculty of

Veterinary Medicine, Utrecht

University, Yalelaan 12

NL-3584 CM Utrecht,

the Netherlands.

Accepted for publication 14 August 1998

Matrix metalloproteinases (MMPs) are essential for normal matrix turnover and play a key part in pathological conditions like osteoarthritis (OA). Matrix turnover in adult articular cartilage is extremely slow. In young individuals metabolism will have to be maintained at a substantially higher level to allow for growth and remodelling. ${ }^{1-3}$ Whether this higher turnover is also reflected by higher MMP activity in synovial fluid (SF) has not been investigated.

$\mathrm{OA}$ is a common and serious problem in horses. ${ }^{4}$ It is generally accepted that marked articular cartilage degeneration can be present despite normal radiographic appearance of the joint. ${ }^{3}$ Therefore, biochemical markers of early articular cartilage degeneration would provide clinicians with a useful tool to assess the current status of the cartilage. Clegg et al demonstrated that both MMP-2 and 9 activities were significantly increased in equine SF from aseptic and septic joint disorders, ${ }^{5}$ but did not specifically focus on OA.

Osteochondrosis (OC) is a disturbance of the process of enchondral ossification, which is very common in juvenile horses. ${ }^{6}$ Induction of OC-like lesions by copper deficiency resulted in increased expression of gelatinase A (MMP-2) in cartilage, which was related to the damage to the matrix structure as it is seen in OC. However, MMP activities in SF were not determined. ${ }^{7}$ Clegg et al found increased activities of MMP-2 and 9 in the SF of horses suffering from aseptic joint disorders including $\mathrm{OC}^{5}$ but did not take into account the age of the horses. Therefore, the increased MMP activities may have resulted from the pathologic condition, or the (young) age of these patients.

Recently, the general MMP substrate TNO211 was developed for measurements of active MMPs in $\mathrm{SF}^{8}$ In the present study this assay was used to measure MMP activity in the $\mathrm{SF}$ of normal juvenile and adult horses to determine whether there is an age effect, and in the SF of joints affected by either OA or OC.

\section{Methods}

EXPERIMENTAL DESIGN

Age

Metacarpophalangeal joint: SF from normal metacarpophalangeal joints of 14 adult horses (mean age 13.4 years, range 4-30) and of 28 juvenile horses (5 months, $\mathrm{n}=14$ and 11 months, $n=14$ ) were obtained within four hours after death and frozen at $-20^{\circ} \mathrm{C}$ until further analysis. Previous studies showed no effect of collection time (up to 12 hours) on MMP activity (unpublished results).

Tarsocrural joint: SF was collected from 11 normal tarsocrural joints of foals of 5 months of age and from seven normal tarsocrural joints of foals of 11 months of age.

\section{Osteoarthritis}

SF of 22 horses (mean age 11.3 years, range 4-23; age matched with the above mentioned control animals; $\mathrm{p}>0.05$ ), with macroscopic evidence of OA (criteria: cartilage thinning, striae formation, fibrillation and erosions of the cartilage $^{9}$ ), were sampled. 

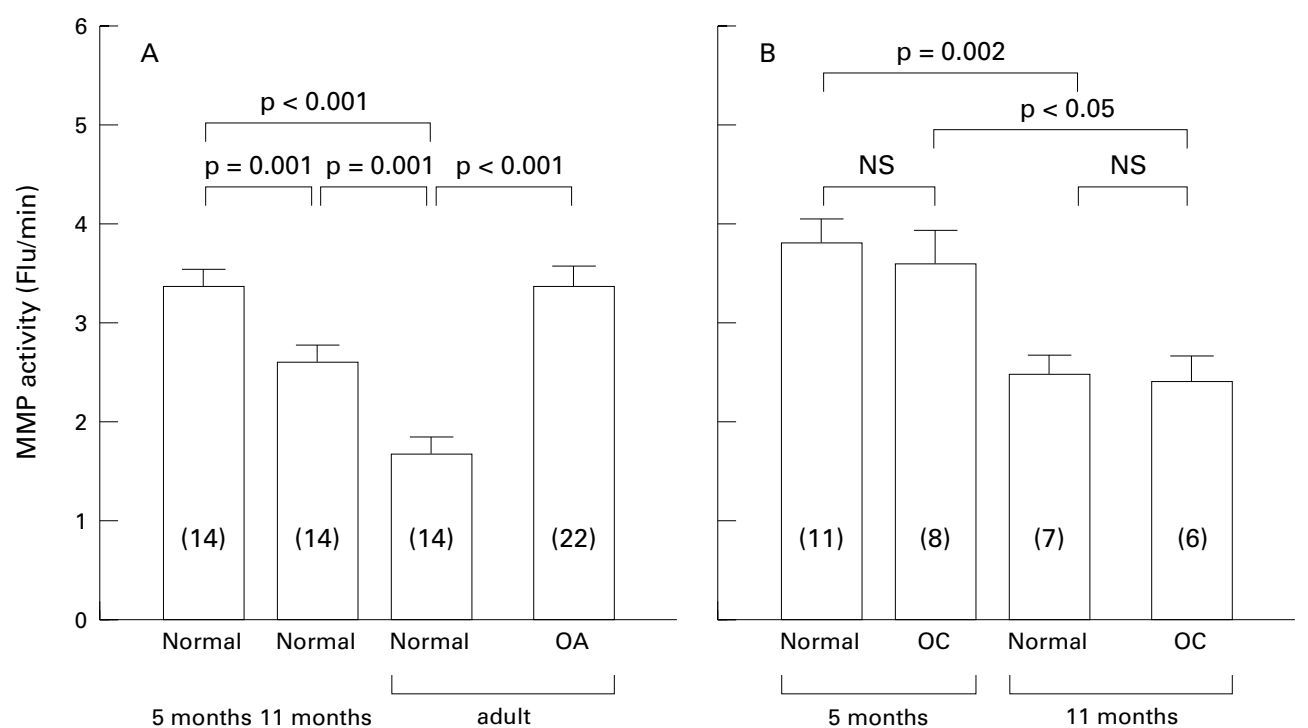

Figure 1 (A) MMP activity (mean (SEM)) in SF of normal juvenile, normal adult, and osteoarthritic equine metacarpophalangeal joints. (B) MMP activity (mean (SEM)) in SF of normal juvenile and osteochondrotic equine tarsocrural joints.

Osteochondrosis

Fourteen joints from foals aged 5 months $(n=8)$ and 11 months $(n=6)$, which were diagnosed osteochondrotic based on radiological, macroscopic, and histological data, were used. MMP activity in SF of the diseased joints was compared with age matched control joints ( 5 months, $\mathrm{n}=11$ and 11 months, $\mathrm{n}=7$ ) without any signs of OC.

MATRIX METALLOPROTEINASES ASSAY

MMP activity in 20-fold diluted SF was measured with a fluorometric assay as described elsewhere and expressed as an increase in fluorescence per minute $(\Delta \mathrm{flu} / \mathrm{min}){ }^{8}$

The used fluorogenic substrate TNO211 (Dabcyl-Gaba-Pro-Gln-Gly-Leu-Glu(EDANS) -Ala-Lys- $\mathrm{NH}_{2}$ ) represents a general MMP substrate: the catalytic effiencies $\left(\mathrm{k}_{\mathrm{cat}} / \mathrm{K}_{\mathrm{m}}\right.$ in $10^{3}$ $\mathrm{M}^{-1} \mathrm{~s}^{-1}$ at $25^{\circ} \mathrm{C}$ ) are for MMP-1, 19; MMP-2, 230; MMP-3, 4; MMP-7, 9, MMP-8, 29; MMP-9, 139; MMP-13, 550, and MMP-14, 147. No conversion of TNO211 by non-MMPs like plasmin, urokinase type plasminogen activator (uPA), or elastase could be detected. ${ }^{8} \mathrm{MMPs}$ captured by alpha-2-macroglobulin can still convert the substrate, whereas TIMP completely inhibits measured MMP activity.

STATISTICAL ANALYSIS

Data were of a normal distribution and with equal variance and presented as the mean (SEM). Differences between groups were tested by a Student's $t$ test. Correlation with age was tested by a Pearson's correlation test. Significance level was set at $\mathrm{p}<0.05$.

\section{Results}

AGE

Metacarpophalangeal joint

MMP activity in normal adult metacarpophalangeal joints $(1.7 \Delta \mathrm{Flu} / \mathrm{min}$; average age 13 years) was almost half of that found in normal juvenile metacarpophalangeal joints (3.0 $\Delta$ Flu/min; age $5-11$ months; $\mathrm{p}<0.001)$. The youngest foals (5 months: $3.5 \Delta \mathrm{Flu} / \mathrm{min}$ ) showed the highest MMP activity (11 months: $2.6 \Delta \mathrm{Flu} / \mathrm{min} ; \mathrm{p}<0.01$; fig $1 \mathrm{~A}$ ). In the adult group no significant correlation between MMP activity and the age of the horse (age range 4-30 years) could be found.

\section{Tarsocrural joint}

MMP activity of 5 month old foals $(3.8 \Delta$ Flu/ $\mathrm{min}$ ) in tarsocrural joints was also significantly higher than in foals of 11 months (2.5 $\Delta$ Flu/min; $\mathrm{p}<0.01$; fig $1 \mathrm{~B})$. MMP activity did not differ significantly between SF from the tarsocrural joints and from the metacarpophalangeal joints of foals ( $p>0.05$, both for 5 month and 11 month age groups).

\section{OSTEOARTHRITIS}

MMP activity in SF of osteoarthritic adult metacarpophalangeal joints $(3.4 \Delta \mathrm{Flu} / \mathrm{min})$ was significantly higher than in the SF of normal adult metacarpophalangeal joints (1.7 $\Delta$ Flu/min; $\mathrm{p}<0.001$; fig $1 \mathrm{~A}$ ).

\section{OSTEOCHONDROSIS}

Figure $1 \mathrm{~B}$ shows the levels of active MMP in $\mathrm{SF}$ from the normal juvenile tarsocrural joints and the osteochondrotic juvenile tarsocrural joints. MMP activity of OC joints of the 5 month old foals $(3.6 \Delta \mathrm{Flu} / \mathrm{min})$ did not differ significantly from normal joints of 5 month old foals $(3.8 \Delta \mathrm{Flu} / \mathrm{min})$. Also, there was no significant difference in MMP activity in SF from the OC joints of 11 month old foals (2.4 $\Delta \mathrm{Flu} / \mathrm{min})$ and the normal joints of 11 month old foals (2.5 $\Delta \mathrm{Flu} / \mathrm{min})$.

\section{Discussion}

At each stage of normal growth, development, and maturation, the relative rates of matrix synthesis and degradation are balanced. At young age greater tissue turnover is required to result in net growth. In pathological conditions an imbalance in synthesis and degradation leads to net tissue degradation. The important 
role of MMPs in tissue turnover is generally accepted. ${ }^{10}$ The results of this study show that MMP activity in SF is significantly higher in immature joints than in mature joints $(p<0.001)$, indicative of a higher matrix turnover in juvenile horses. These differences were consistently found in both the metacarpophalangeal and tarsocrural joints. These results emphasise the need for the use of age matched controls when studying any pathological condition, especially when conditions of growing individuals are concerned like in OC.

Early diagnosis of OA is a major problem, both in human and veterinary medicine. ${ }^{311}$ Previously we showed that MMP activity in SF from patients with rheumatoid arthritis or OA was significantly increased as compared with $\mathrm{SF}$ from control joints. ${ }^{8}$ In the equine, Clegg et al showed that MMP-2 and MMP-9 activities are increased significantly in a broad range of aseptic joint diseases. ${ }^{5}$ In this study a significant difference in overall MMP activity was found between normal and OA joints. These results are similar to those found in humans ${ }^{8}$ and specify the findings by Clegg et al. ${ }^{5}$

Any member of the MMP family present in the synovial fluid can contribute to the measured conversion of substrate TNO211. In particular, the gelatinases MMP-2 and -9 , as well as the collagenase MMP-13 and, to a lesser extent, MMP-14 can efficiently degrade TNO211. However, other MMPs like MMP-1 and -3 , having lower affinities for TNO211, could still be major contributors if their concentration in SF is much higher than that, for example, of MMP-13. To investigate which member of the MMP family plays a pivotal part in physiological and pathological turnover of the cartilage, the required specific assays are being developed at present. It should be realised that the presence of TIMPs in the cartilage matrix may affect the effect on the matrix of MMPs measured in the SF as they can form a line of defence against penetrating MMPs from the SF. Also, a high concentration of alpha-2-macroglubulin in the SF may result in an overestimation of MMPs available to degrade the cartilage matrix.
No increase in MMP activity was found in osteochondrotic joints. This result is not in agreement with the data from Clegg et al who found a rise in MMP-2 and -9 activities in all aseptic joint disorders, including OC. ${ }^{5}$ Inasmuch as Clegg et al did not specify the age of the animals, their finding of increased gelatinase activities may result from the fact that the age of their OC group was substantially lower than that of the control group.

It is hypothesised that MMP activity in equine $\mathrm{SF}$ reflects, at least to a certain extent, metabolism taking place in the matrix. In the juvenile horse high MMP activity is indicative of physiological tissue remodelling during growth. In the adult horse MMP activity is low. Increased MMP activity in SF of mature horses is likely to indicate matrix destruction. Hence, MMP activity may be a useful tool in diagnostic, therapeutic or prognostic studies in horses suspected of OA, provided that age is taken into account. In osteochondrosis, there is no rise in general MMP activity, indicating no widespread MMP mediated cartilage damage.

1 Murphy G, Hembry RM, Hughes CE, Fosang AJ, Hardingham TE. Role and regulation of metalloproteinases in connective tissue turnover. Biochem Soc Trans in connective tisst.

2 Todhunter RJ. Anatomy and physiology of synovial joints. In: McIlwraith CW, Trotter GW, eds. Foint disease in the In: Mcllwraith CW, Trotter GW, eds. Fo

3 Lohmander LS, Lark MW, Dahlberg L, Walakovits LA, Lohmander LS, Lark MW, Dahlberg L, Walakovits LA,
Roos H. Cartilage matrix metabolism in osteoarthritis: markers in synovial fluid, serum, and urine. Clin Biochem 992;25:167-74.

4 Rossdale PD, Hopes R, Wingfield-Digby NJ, Offord K. Epidemiological study of wastage among racehorses 1982 and 1983. Vet Rec 1985;116:66-9.

5 Clegg PD, Coughlan AR, Riggs CM, Carter SD. Matrix metalloproteinases 2 and 9 in equine synovial fluids. Equine Vet J 1997;29:343-8.

6 Hurtig MB, Pool RR. Pathogenesis of equine osteochondrosis. In: McIlwraith CW, Trotter GW eds. Foint disease in the horse.Philadelphia: Saunders, 1996:335-58.

7 Hurtig M, Green SL, Dobson H. Correlative study of defective cartilage and bone growth in foals fed a low-copper diet. Equine Vet J Suppl 1993;19:66-73.

8 Beekman B, Drijfhout JW, Bloemhoff W, Ronday HK, Tak PP, TeKoppele JM. Convenient fluorometric assay for $\mathrm{PP}$, TeKoppele JM. Convenient fluorometric assay for
matrix metalloproteinase activity and its application in biomatrix metalloproteinase activity and its applicatic

9 McIlwraith CW. General pathobiology of the joint and Mcllwraith CW. General pathobiology of the joint and
response to injury. In: McIlwraith CW, Trotter GW, eds. Foint disease in the horse. Philadelphia: Saunders, 1996:40-70.

10 Dieppe P. Osteoarthritis: clinical and research perspective. Br J Rheumatol 1991;30 (suppl 1):1-4.

11 Rørvik AM, Grøndahl AM. Markers of osteoarthritis: A review of the literature. Vet Surg 1995;24:255-62. 